\title{
ON THE DYNAMICS OF SEMIMARTINGALES WITH TWO REFLECTING BARRIERS
}

\author{
MATS PIHLSGÅRD, ${ }^{*}$ Lund University \\ PETER W. GLYNN, ${ }^{* *}$ Stanford University
}

\begin{abstract}
We consider a semimartingale $X$ which is reflected at an upper barrier $T$ and a lower barrier $S$, where $S$ and $T$ are also semimartingales such that $T$ is bounded away from $S$. First, we present an explicit construction of the reflected process. Then we derive a relationship in terms of stochastic integrals linking the reflected process and the local times at the respective barriers to $X, S$, and $T$. This result reveals the fundamental structural properties of the reflection mechanism. We also present a few results showing how the general relationship simplifies under additional assumptions on $X, S$, and $T$, e.g. if we take $X, S$, and $T$ to be independent martingales (which satisfy some extra technical conditions).
\end{abstract}

Keywords: Skorokhod problem; reflection; semimartingale; Lévy process; martingale; stochastic integration

2010 Mathematics Subject Classification: Primary 60G44; 60G51; 60H05

Secondary $60 \mathrm{G} 17$

\section{Introduction}

Lately, two-sided reflected stochastic processes have received attention by many authors. The study of such objects may be motivated from an applied as well as a purely theoretical perspective. Possible applications are buffer systems, finite capacity dam models, and queueing systems (see, e.g. [4], [7], [9], [11], [16], [18], and [21]) and a variety of telecommunication models (see, e.g. [11], [14], and [22]). Two main examples in this area are the following.

- Two-sided reflected random walks (reflected in 0 and $b>0$ ). The reflected process $V$ is given by the recursion

$$
V_{n+1}=\min \left[b, \max \left(0, V_{n}+Y_{n}\right)\right],
$$

where $Y_{1}, Y_{2}, \ldots$ are random variables.

- In analogy with the first example, a process $X$ (with $X(0)=0$ ) with two-sided reflection at 0 and $b$. The reflected process $V$ is constructed as

$$
V(t)=V(0)+X(t)+L(t)-U(t),
$$

where $L$ and $U$ are the local times at 0 and $b$, respectively. The process $(V, L, U)$ is given as a solution to the Skorokhod problem corresponding to the reflection, which is described in, e.g. [6].

Received 17 April 2012; revision received 4 December 2012.

* Postal address: Clinical Research Centre, Lund University, Building 28, Floor 13, Jan Waldenströms gata 35, 20502 Malmö, Sweden. Email address: mats.pihlsgard@med.lu.se

** Postal address: Management Science and Engineering, Stanford University, Stanford, CA 94305-4121, USA. Email address: glynn@stanford.edu 
Taking the setup described in (1), with $X$ a Lévy process, as a starting point, one may proceed to more sophisticated models, e.g. Markov modulated input (see [6]), dynamic barriers (see, e.g. [13] and [17]), and combinations of the aforementioned generalizations (see, e.g. [10]).

The main themes of earlier work are the identification of $\ell=\mathbb{E} U(1)$ (under stationarity) (see [6], where $b$ is fixed, and [17], where the upper barrier is dynamic), asymptotics of $\ell$ as $\mathbb{R}^{+} \ni b \rightarrow \infty$ under different assumptions on $X$ (see, e.g. [1], [2], [6], and [17]), and the Markov transition kernel of $V$ (if relevant) and the corresponding stationary distribution (see, e.g. [10]).

In this paper we switch focus. Rather than investigating yet another special case, we seek generality. The goal is to give a concise account of the structural properties of the two-sided reflection, i.e. to give an understanding of the basic mechanisms governing the reflection. In Section 3 we start by constructing the reflected process $V$ in terms of the feeding process $X$ and the barriers (the barriers will be denoted by $S$ and $T$ ); see Proposition 1. After that we prove that the local times $L$ and $U$ exist; see Proposition 2. Then we proceed to the main result, which is given in Theorem 1. This shows the relation between $(V, L, U)$ and the input. In Section 4 we consider reflection under stationary conditions. Many of the results given in earlier work follow from the results presented there in a fairly straightforward way. For example, the main result in [6], which was proven in a very complicated indirect manner (which, by the way, sheds little probabilistic light upon the problem), is an easy consequence of Theorem 2 below; see Example 3. In Section 5 we show that, under certain additional assumptions on $X, S$, and $T$, the general relationship simplifies significantly.

One key point of this paper is that the dynamics of the two-sided reflection are governed by stochastic integrals involving the feeding process and the barriers. Thus, all that is required is that stochastic integration makes sense. Hence, the natural framework is to take $X, S$, and $T$ to be semimartingales.

\section{Preliminaries}

We start with a brief discussion about semimartingales. A standard reference on this topic is [19]. We assume that we are given a probability space $(\Omega, \mathcal{F}, \mathcal{F}(t), \mathbb{P})$ (where the filtration $\mathcal{F}(t)$, as always, satisfies the usual conditions, i.e. it is augmented and right continuous). A stochastic process defined on $(\Omega, \mathcal{F}, \mathcal{F}(t), \mathbb{P})$ is a semimartingale if it is adapted, càdlàg, and admits a decomposition

$$
X(t)=X(0)+N(t)+B(t)
$$

where $N$ is a local martingale and $B$ a process of finite variation on compacts (almost surely (a.s.)) with $N(0)=B(0)=0$. Alternatively, a semimartingale $X$ is a stochastic process for which the stochastic integral

$$
\int H(s) \mathrm{d} X(s)
$$

is well defined for $H$ belonging to a satisfactory rich class of processes (more precisely, the predictable processes; see [19]). In (2), we will in this paper take $H$ to be an adapted process (with respect to $\mathcal{F}(t)$ ) with left-continuous paths with right limits.

The class of semimartingales forms a vector space, is closed under suitable changes of measure, and if $X$ is a semimartingale for the filtration $\mathcal{F}(t)$ and $g(t)$ is a subfiltration of $\mathcal{F}(t)$ such that $X$ is adapted to $g(t)$ then $X$ is a $g(t)$ semimartingale. For more stability properties of semimartingales, we refer the reader to [19]. Furthermore, the class of semimartingales is 
large, containing, e.g. adapted processes with càdlàg paths of finite variation on compacts and Lévy processes.

We denote the class of semimartingales by $\mathbb{S}$. If $f$ is a real-valued function defined on some subset of $\mathbb{R}$, we let $f(t-)=\lim _{s \uparrow t} f(s), f(t+)=\lim _{s \downarrow t} f(s)$ (provided that the limits exist), and $\Delta f(t)=f(t)-f(t-)$. For a set $A$, we let $\mathbf{1}_{A}$ denote the corresponding indicator function. Let $X$ and $Y$ be semimartingales; $[X, X]$ denotes the quadratic variation process of $X,[X, X]^{\mathrm{c}}$ is the continuous part of $[X, X]$, and $[X, Y]$ is the quadratic covariation process (also referred to as the bracket process) of $X$ and $Y$.

\section{Identification of the general relationship}

Let $X, S$, and $T$ be semimartingales on which we impose the restrictions that $X(0)=0$ and $S(t)+\varepsilon<T(t)$ for some $\varepsilon>0$ and all $t \in \mathbb{R}^{+}$. We now introduce the Skorokhod problem that we wish to investigate. Find $(V, L, U)$, with $S(t) \leq V(t) \leq T(t)$ for all $t \in \mathbb{R}^{+}, L$ and $U$ nonnegative (for simplicity, we take $L(0)=U(0)=0$ ), $L(t)$ and $U(t)$ finite for all $t \in \mathbb{R}^{+}$, and $L$ and $U$ nondecreasing and right continuous, such that

$$
V(t)=V(0)+X(t)+L(t)-U(t)
$$

and

$$
\int_{0}^{\infty}(V(s)-S(s)) \mathrm{d} L(s)=0, \quad \int_{0}^{\infty}(T(s)-V(s)) \mathrm{d} U(s)=0 .
$$

We may think of $V$ as the process which results from reflecting $X$ in $S$ and $T$, and of $L$ and $U$ as the local times at $S$ and $T$, i.e. the 'pushing away' from $S$ and $T$, respectively, required to keep $S(t) \leq V(t) \leq T(t)$. The conditions in (4) say that $L$ and $U$ increase only when $V$ is at the respective barrier. Note that this as a constraint on $L$ and $U$, i.e. we cannot use (4) to determine $V$, but it does, however, establish a link between $L, U$, and $V$. In what follows we may assume without loss of generality that $S \equiv 0$ and $T>\varepsilon$. All results shown for this setup can easily be generalized to hold for reflection with a nonconstant lower barrier.

At this point we may wonder about the existence and uniqueness of a solution $(V, L, U)$. We will first construct $V$ in terms of $V(0), X$, and $T$. Then we prove the existence of $L$ and $U$. One can show uniqueness of $(V, L, U)$ in a fashion similar to the proof of Proposition 2.2 of [5, p. 251]; see also [3]. With these results at hand, it is possible to identify $L$ and $U$; see Theorem 1. In the paper by Kruk et al. [15], an explicit solution to the Skorokhod problem on $[0, b]$ is presented. We will use the representation given by (here $\left.X^{\prime}(t)=V(0)+X(t)\right)$

$$
V_{b}(t)=X^{\prime}(t)-\left((V(0)-b)^{+} \wedge \inf _{u \in[0, t]} X^{\prime}(u)\right) \vee \sup _{s \in[0, t]}\left(\left(X^{\prime}(s)-b\right) \wedge \inf _{u \in[s, t]} X^{\prime}(u)\right),
$$

which we, in obvious notation, write as $V_{b}(t)=X^{\prime}(t)-f_{b}(t)$. The next result is simple. Guided by (5), we construct (guess) a function and show that it meets the requirements for the reflected process.

Proposition 1. Let $X^{\prime}(t)=V(0)+X(t)$. Then we may identify $V(t)$ in (3) as

$$
\begin{aligned}
V(t)= & X^{\prime}(t) \\
& -\left((V(0)-T(0))^{+} \wedge \inf _{u \in[0, t]} X^{\prime}(u)\right) \vee \sup _{s \in[0, t]}\left(\left(X^{\prime}(s)-T(s)\right) \wedge \inf _{u \in[s, t]} X^{\prime}(u)\right) .
\end{aligned}
$$


Proof. It is not difficult to see that $V(t)$ satisfies $0 \leq V(t) \leq T(t)$. The modifying term $f$, say, in (6) (defined as $f(t)=X^{\prime}(t)-V(t)$ ) is càdlàg and of bounded variation because $f_{\varepsilon}$ is so. It follows by a slight generalization of the results in [12] that what we need to show is that $f$ can only increase when $T(t)=V(t)$ and can only decrease when $V(t)=0$. Let $I_{f}$ denote the points of increase of $f$, i.e.

$$
I_{f}=\{t \geq 0: \text { there exists } \epsilon>0 \text { such that } f(t-)<f(s), t<s<t+\epsilon\},
$$

where $f(0-)$ should be interpreted as 0 . Take $t^{\prime} \in I_{f}$ and $t^{\prime}<v$ such that $f(t)>f\left(t^{\prime}-\right)$ for $t^{\prime}<t<v$. We note that $f\left(t^{\prime}-\right) \geq(V(0)-T(0))^{+} \wedge \inf _{u \in[0, t]} X^{\prime}(u)$, so

$$
f(t)=\sup _{s \in[0, t]}\left(\left(X^{\prime}(s)-T(s)\right) \wedge \inf _{u \in[s, t]} X^{\prime}(u)\right) .
$$

Also,

$$
f\left(t^{\prime}-\right) \geq\left(X^{\prime}(s)-T(s)\right) \wedge \inf _{u \in[s, t]} X^{\prime}(u), \quad s \in\left[0, t^{\prime}\right) .
$$

The combination of (7) and (8) implies that

$$
f(t)=\sup _{s \in\left[t^{\prime}, t\right]}\left(\left(X^{\prime}(s)-T(s)\right) \wedge \inf _{u \in[s, t]} X^{\prime}(u)\right) .
$$

It follows by the right continuity of $X$ and $T$ that $f\left(t^{\prime}\right)=f\left(t^{\prime}+\right)=X^{\prime}\left(t^{\prime}\right)-T\left(t^{\prime}\right)$. Thus, $V\left(t^{\prime}\right)=X^{\prime}\left(t^{\prime}\right)-\left(X^{\prime}\left(t^{\prime}\right)-T\left(t^{\prime}\right)\right)=T\left(t^{\prime}\right)$. The details for the lower barrier are similar.

Proposition 2. The solution $(V, L, U)$ exists.

Proof. The process $V$ is constructed in Proposition 1. We assume without loss of generality that $V(0)=0$. Recall that, by assumption, $T(u)>\varepsilon$ for all $u \geq 0$. We construct $L$ and $U$ by alternating between the two more standard one-sided reflection operators corresponding to downward reflection at $T$ and upward reflection at 0 according to the epochs where 0 or $T$ is hit. All we have to do to complete the proof is to show that $L(t), U(t)<\infty$ for all $t \in \mathbb{R}^{+}$, since, by construction, $L$ and $U$ will then satisfy the other conditions in the formulation of the Skorokhod problem. We define the cycles of $V$ to be the shortest time segments during which $V$ moves from 0 to the upper barrier $T$ and back to 0 . Each cycle makes a finite contribution to $L$ and $U$. Let $t>0$. By applying Lemma 1 of [8, p. 122] to the restriction of $V$ to [0, $t$, we see that there exists $a>0$ such that all cycles completed in $[0, t]$ are longer than $a$. This means that there does not exist an accumulation point of cycles in $[0, t]$ and, thus, $L(t), U(t)<\infty$.

Remark 1. In the first version of the paper we only required that $T(t)>S(t)$, but then a solution $(V, L, U)$ may fail to exist. This was brought to our attention by an anonymous referee who gave an example with $X(t)<\infty$ for all $t, S(t)=0$, and $T(t)>0$ such that there exists a point $u<\infty$ with $L(u-)=U(u-)=\infty$.

The next two results follow from the conditions given in the Skorokhod problem.

Proposition 3. Let $V, L$, and $U$ be as in (3). Then $V, L$, and $U$ are semimartingales.

Proof. Since $L$ and $U$ are càdlàg and increasing (thus of bounded variation), it follows that they are semimartingales. Semimartingales form a vector space, so we are done.

Proposition 4. It holds that $[V, V]^{\mathrm{c}}=[X, X]^{\mathrm{c}}$. 
Proof. $L-U$ is càdlàg and of bounded variation, and it follows by Theorem 26 of [19, p. 71] that $[L-U, L-U]^{\mathrm{c}}=0$, which is well known to imply that $[X, L-U]^{\mathrm{c}}=0$; see, e.g. Theorem 28 of [19, p. 75]. The claim now follows from

$[V, V]^{\mathrm{c}}=[X+L-U, X+L-U]^{\mathrm{c}}=[X, X]^{\mathrm{c}}+[L-U, L-U]^{\mathrm{c}}+2[X, L-U]^{\mathrm{c}}=[X, X]^{\mathrm{c}}$.

To make things slightly more formal, we introduce an operator $R$ corresponding to the reflection defined by (3) and (4). In view of Proposition 3 we have $R: \mathbb{S}^{2} \rightarrow \mathbb{S}^{3}$. We now proceed straight to the main result, which establishes the link between $(L, U)$ and $(X, T)$. We choose to mainly focus on the local time $U$, by partly eliminating $L$, but it should be obvious how to obtain the corresponding results for $L$. The processes $T, V$, and $(T-V)$ are nonnegative, càdlàg, and $L(t), U(t)<\infty$ for all $t>0$, which together with (4) implies that $\int_{0+}^{t} T(s) \mathrm{d} U(s)$ and $\int_{0+}^{t} V(s) \mathrm{d} U(s)$ are finite and equal and $\int_{0+}^{t} V(s) \mathrm{d} L(s)=0$ for all $t>0$. We remark that $\int_{0+}^{t} T(s) \mathrm{d} U(s)$ has to be rewritten according to

$$
\begin{aligned}
\int_{0+}^{t} T(s) \mathrm{d} U(s) & =\int_{0+}^{t} T(s-) \mathrm{d} U(s)+\int_{0+}^{t} \Delta T(s) \mathrm{d} U(s) \\
& =\int_{0+}^{t} T(s-) \mathrm{d} U(s)+\sum_{0<s \leq t} \Delta T(s) \Delta U(s) \\
& =\int_{0+}^{t} T(s-) \mathrm{d} U(s)+[T, U](t)
\end{aligned}
$$

in order to be meaningful (in the last step we again used Theorem 28 of [19, p. 75], which is applicable because $U$ is of bounded variation). If we perform integration by parts in (9), we obtain the identity

$$
\int_{0+}^{t} T(s) \mathrm{d} U(s)=T U(t)-\int_{0+}^{t} U(s-) \mathrm{d} T(s) .
$$

Theorem 1. Let $(V, L, U)=R((X, T))$. Then the structural relationship

$$
2 T U(t)=V(0)^{2}-V(t)^{2}+2 \int_{0+}^{t}(V(s-) \mathrm{d} X(s)+U(s-) \mathrm{d} T(s))+[X, X]^{\mathrm{c}}(t)+J_{R}(t)
$$

holds, where $J_{R}$ is pure jump of bounded variation with

$$
\begin{aligned}
J_{R}(t) & =\sum_{0<s \leq t}\left(-2 \Delta V(s) \Delta L(s)+2 \Delta V(s) \Delta U(s)+(\Delta V(s))^{2}\right) \\
& =\sum_{0<s \leq t}\left((\Delta X(s))^{2}-(\Delta L(s))^{2}-(\Delta U(s))^{2}\right) .
\end{aligned}
$$

Proof. Plainly, by the definition of the quadratic variation process $[V, V]$ and Proposition 4 ,

$$
V(t)^{2}-V(0)^{2}-2 \int_{0^{+}}^{t} V(s-) \mathrm{d} V(s)=[V, V](t)=[X, X]^{\mathrm{c}}(t)+\sum_{0<s \leq t}(\Delta V(s))^{2} .
$$

Furthermore,

$$
\mathrm{d} V(t)=\mathrm{d} X(t)+\mathrm{d} L(t)-\mathrm{d} U(t) \quad \text { and } \quad V(s-)=V(s)-\Delta V(s),
$$


so (3) yields

$$
\begin{aligned}
\int_{0^{+}}^{t} V(s-) \mathrm{d} V(s)= & \int_{0^{+}}^{t} V(s-) \mathrm{d} X(s)+\int_{0^{+}}^{t}(V(s)-\Delta V(s)) \mathrm{d} L(s) \\
& -\int_{0^{+}}^{t}(V(s)-\Delta V(s)) \mathrm{d} U(s) \\
= & \int_{0^{+}}^{t} V(s-) \mathrm{d} X(s)-\int_{0^{+}}^{t} \Delta V(s) \mathrm{d} L(s)-\int_{0^{+}}^{t} T(s) \mathrm{d} U(s) \\
& +\int_{0^{+}}^{t} \Delta V(s) \mathrm{d} U(s) \\
= & \int_{0^{+}}^{t} V(s-) \mathrm{d} X(s)-\sum_{0<s \leq t} \Delta V(s) \Delta L(s)-\int_{0^{+}}^{t} T(s) \mathrm{d} U(s) \\
& +\sum_{0<s \leq t} \Delta V(s) \Delta U(s) .
\end{aligned}
$$

Equation (11) and the first representation in (12) follow by combining (10), (13), and (14). The second equality in (12) follows from the trivial identites

$$
\begin{aligned}
(\Delta X(s))^{2}= & (\Delta V(s)+\Delta U(s)-\Delta L(s))^{2} \\
= & (\Delta V(s))^{2}+(\Delta L(s))^{2}+(\Delta U(s))^{2}+2 \Delta V(s) \Delta U(s) \\
& -2 \Delta V(s) \Delta L(s)-2 \Delta L(s) \Delta U(s)
\end{aligned}
$$

and the condition $T(t)>\varepsilon$, which implies that $\Delta L(s) \Delta U(s)=0$. Finally, if we write

$$
\begin{aligned}
& \sum_{0<s \leq t}\left((\Delta X(s))^{2}-(\Delta L(s))^{2}-(\Delta U(s))^{2}\right) \\
& \quad=\sum_{0<s \leq t}(\Delta X(s))^{2}-\sum_{0<s \leq t}\left((\Delta L(s))^{2}+(\Delta U(s))^{2}\right),
\end{aligned}
$$

and note that

$$
\sum_{0<s \leq t}\left((\Delta X(s))^{2} \leq[X, X](t) \text { and } \sum_{0<s \leq t}\left((\Delta L(s))^{2}+(\Delta U(s))^{2}\right) \leq L(t)^{2}+U(t)^{2},\right.
$$

we see that $J_{R}(t)$ is the difference between two increasing processes which are finite for each $t$, and thus of bounded variation.

Example 1. Let $X$ be a continuous semimartingale. We look at the recursion where $T(t)=$ $\varepsilon+U(t)$, i.e. $(V, L, U)=R(X, U+\varepsilon)$. Then it follows, by (11) (clearly, $J_{R}(t)=0$ ) and straightforward calculus, that $U(t)$ satisfies the equation

$$
U(t)^{2}+2 \varepsilon U(t)=V(0)^{2}-V(t)^{2}+2 \int_{0+}^{t} V(s-) \mathrm{d} X(s)+[X, X]^{\mathrm{c}}(t) .
$$

In the representation of $J_{R}$ it should be clear that the part $(\Delta U(s))^{2}$ is the most complicated, since in the general case it may be generated by both $\Delta X$ and $\Delta T$. This is not the case for $(\Delta L(s))^{2}$, since we assume that $T>\varepsilon$. It is plain to see that

$$
\Delta U(s)=\max (0,(\Delta X(s)-\Delta T(s))-T(s-)+V(s-)),
$$

so that the sum in (12) is over the set $\xi_{X T}(t)=\{s \in(0, t]: \Delta X(s) \neq 0$ or $\Delta T(s)<0\}$. We now present a few results which show how the result presented in Theorem 1 simplifies 
under different assumptions on $(X, T)$. Let $\xi_{X}(t)=\{s \in(0, t]: \Delta X(s) \neq 0\}$ and $\xi_{T}(t)=$ $\{s \in(0, t]: \Delta T(s)<0\}$.

Proposition 5. If $T$ is downwards skip-free then $J_{R}$ has the representation

$$
J_{R}(t)=\sum_{s \in \xi_{X}(t)} \varphi(T(s), V(s-), \Delta X(s))
$$

where

$$
\varphi(v, x, y)= \begin{cases}-\left(x^{2}+2 x y\right) & \text { if } y \leq-x, \\ y^{2} & \text { if }-x<y<v-x, \\ 2 y(v-x)-(v-x)^{2} & \text { if } y \geq v-x .\end{cases}
$$

In particular, this means that $J_{R}(t)$ is nondecreasing.

Proof. Equation (15) follows directly from (12) (and some easy algebra) if we note that if $T$ is downwards skip-free then $\xi_{X T}(t)=\xi_{X}(t)$ for each $t$ and

$$
\begin{gathered}
\Delta L(s)=\max (0,-(\Delta X(s)+V(s-))), \\
\Delta U(s)=\max (0, \Delta X(s)+V(s-)-T(s)) .
\end{gathered}
$$

The next proposition shows how to express $J_{R}$ under the assumption that the bracket process $[X, T]$ is continuous.

Proposition 6. Suppose that $X$ and $T$ are such that $[X, T]$ is a.s. continuous. Then, a.s., for each $t, \xi_{X}(t)$ and $\xi_{T}(t)$ are disjoint and

$$
\begin{aligned}
J_{R}(t)= & \sum_{s \in \xi_{X}(t)} \varphi(T(s-), V(s-), \Delta X(s)) \\
& -\sum_{s \in \xi_{T}(t)}(\max (0,(-\Delta T(s)-T(s-)+V(s-))))^{2} .
\end{aligned}
$$

Proof. From Theorem 23 of [19, p. 68], we have $\Delta[X, T](s)=\Delta X(s) \Delta T(s)$, so the assumption yields $\Delta X(s) \Delta T(s)=0$ a.s. and the first claim follows. Equation (16) follows easily (the first part is shown in the same way as in the proof of Proposition 5) if we note that, for $s \in \xi_{X}(t), \Delta U(s)=\max (0, \Delta X(s)+V(s-)-T(s-))$, and, for $s \in \xi_{T}(t), \Delta U(s)=$ $\max (0, V(s-)-T(s-)-\Delta T(s)), \Delta X(s)=\Delta L(s)=0$.

Remark 2. Of course, one could replace the condition in Proposition 6 ( $[X, T]$ continuous) by $\Delta X(s) \Delta T(s)=0$. However, in our opinion, when operating on semimartingales, it is more natural (at least more traditional) to express the condition as a restriction on $[X, T]$.

The next result shows that if the upper barrier is constant then it is possible to identify $U$ in terms of $X$ and the barrier level.

Corollary 1. If $T=b$, where $b>0$ is a real constant, then it holds that

$$
U(t)=(2 b)^{-1}\left(V(0)^{2}-V(t)^{2}+2 \int_{0+}^{t} V(s-) \mathrm{d} X(s)+[X, X]^{\mathrm{c}}(t)+J_{R}(t)\right),
$$

where

$$
J_{R}(t)=\sum_{0<s \leq t} \varphi(b, V(s-), \Delta X(s))
$$

Proof. Equations (17) and (18) are immediate consequences of Theorem 1 and Proposition 5 (or Proposition 6). 


\section{Reflection under stationary conditions}

In much of what has been written on reflected (Lévy) processes, it is assumed that $V$ is stationary. The goal is then typically to calculate a 'boundary measure' quantity via 'interior measure' quantities; e.g. to find an expression for $\ell=\mathbb{E} U(1)$ in terms of the stationary measure of $V$, which we denote by $\pi$, and the Lévy measure of $X$. The main motivation in, e.g. [6] for doing this is that $\pi([x, b])$ is accessible (at least in principle) when $T=b>0$ is constant. In fact, it equals the probability of the event that the unrestricted Lévy process $X$ makes its first exit from $[x-b, x)$ to the right. We include this section to show that some results proven in earlier work follow easily from and can be extended by the more general theory developed in the present paper. Note that, when $T$ is not constant, $\mathbb{E} U(t)$ is not accessible in general; however, $\mathbb{E} \int_{0+}^{t} T(s) \mathrm{d} U(s)$ is accessible.

When the feeding process as well as the boundaries are taken to be general semimartingales, it is not possible to guarantee the existence of a stationary distribution of $V$. In order to be able to do this, we have to make strong additional assumptions about $X$ and $T$. In the current section, $X$ is a Lévy process and $T \leq b$ for some constant $b$.

Proposition 7. Suppose that $T=b>0$ and that $X$ is a Lévy process. Then there exists an asymptotic (and thus stationary) distribution $\pi$ on $[0, b]$ for $V$.

Proof. The assertion is clearly true if $X$ has monotone sample paths, so we assume that this is not the case. The existence of an asymptotic distribution follows if we can show that $V$ is regenerative with nonlattice cycle length distribution with finite mean (see [5, Theorem 3.1, p. 170]). We assume without loss of generality that $V(0)=0$. If we let $\tau(1)=0$ and, for $k \geq 2, \tau(k)=\inf \left\{t>\tau(k-1): V(t)=0, \sup _{\tau(k-1)<s \leq t} V(s)>b / 2\right\}$, we may take the cycles to be $(V(s), s \in[\tau(k-1), \tau(k)))$. Clearly, the cycle length distribution is nonlattice. Choose $d>0$ such that $\mathbb{P}(X(d)>b / 2)>0$ (this is possible to do; see Theorem 24.10 of [20, p. 152]). Let $\tau=\min \{n>0: X(n d)-X((n-1) d)>b / 2\}$. Then, by the Markov property of $X$ and a geometric trial argument, it follows that $\mathbb{E} \tau<\infty$. This proves that the mean of the first part of the cycle (from 0 to above $b / 2$ ) is finite. In a similar way we can prove that the second part of the cycle (back to 0 ) has finite mean.

As the next example shows, there are cases with $T$ not constant where we have a stationary distribution of $(T, V)$.

Example 2. Let $0<\varepsilon<b$. It follows from [17] that if we take $X$ to be a Lévy process and the barrier $T$ to be of the form

$$
T(t)=f(t+U),
$$

where $f$ is a periodic function with $\varepsilon \leq f \leq b$ and period $\Omega$, and $U$ is a random variable with uniform distribution on $[0, \Omega]$, then there exists a stationary measure on $[\varepsilon, b]^{2}$ for $(T, V)$.

The next result tells us how to express expectations (under stationary conditions) in (11) in terms of the characteristic triplet of $X$ and the stationary distribution $\pi$ of $(T, V)$.

Proposition 8. Suppose that $X$ is a Lévy process with characteristic triplet $(\mu, \sigma, v)$ and $\mathbb{E}|X(1)|<\infty$.

(i) Let

$$
I(t)=\int_{0+}^{t} V(s-) \mathrm{d} X(s) .
$$

If $(T(0), V(0)) \stackrel{\mathrm{D}}{=} \pi$ it holds that $\mathbb{E} I(t)=t \mathbb{E} V(0) \mathbb{E} X(1)$. 
(ii) If $(T(0), V(0)) \stackrel{\mathrm{D}}{=} \pi$ and $T$ is a downwards skip-free, stationary semimartingale, with $[X, T]$ a.s. continuous, then

$$
\mathbb{E} J_{R}(t)=t \int_{0}^{b} \int_{0}^{b} \pi(\mathrm{d} x, \mathrm{~d} y) \int_{-\infty}^{\infty} \varphi(x, y, z) v(\mathrm{~d} z) .
$$

Proof. Let $\tilde{X}(t)=X(t)-\sum_{0<s \leq t} \Delta X(s) \mathbf{1}_{\{|\Delta X(s)| \geq 1\}}$, so that

$$
I(t)=\int_{0+}^{t} V(s-) \mathrm{d} \tilde{X}(s)+\sum_{0<s \leq t} V(s-) \Delta X(s) \mathbf{1}_{\{|\Delta X(s)| \geq 1\}} .
$$

We let $\tilde{Y}(t)=\tilde{X}(t)-t \mathbb{E} \tilde{X}(1)$. Then $\tilde{Y}$ is a martingale (and thus a local martingale) and it follows by Theorem 29 of $\left[19\right.$, p. 128] that $J(t)=\int_{0+}^{t} V(s-) \mathrm{d} \tilde{Y}(s)$ is also a local martingale. Theorem 29 of $[19$, p. 75$]$ tells us that

$$
\begin{aligned}
{[J, J](t) } & =\int_{0+}^{t} V(s-)^{2} \mathrm{~d}[\tilde{Y}, \tilde{Y}](s) \\
& =\int_{0+}^{t} V(s-)^{2} \mathrm{~d}[\tilde{X}, \tilde{X}](s) \leq b^{2}[\tilde{X}, \tilde{X}](t) \\
& =b^{2}\left(\sigma^{2} t+\sum_{0<s \leq t}(\Delta X(s))^{2} \mathbf{1}_{\{|\Delta X(s)|<1\})},\right.
\end{aligned}
$$

and it follows that $\mathbb{E}[J, J](t)<\infty$ for all $t \geq 0$, which implies that $J$ is a martingale; see Corollary 3 of $[19$, p. 73$]$. Then $\mathbb{E} J(t)=\mathbb{E} J(0)=0$, and, thus,

$$
\mathbb{E} \int_{0+}^{t} V(s-) \mathrm{d} \tilde{X}(s)=\mathbb{E} \int_{0+}^{t} V(s-) \mathbb{E} \tilde{X}(1) \mathrm{d} s=t \mathbb{E} V(0) \mathbb{E} \tilde{X}(1) .
$$

Furthermore, since $\sum_{0<s \leq t} \Delta X(s) \mathbf{1}_{\{|\Delta X(s)| \geq 1\}}$ is a compound Poisson process and $V(s-)$ is independent of $\Delta X(s)$, we obtain

$$
\mathbb{E} \sum_{0<s \leq t} V(s-) \Delta X(s) \mathbf{1}_{\{|\Delta X(s)| \geq 1\}}=t \mathbb{E} V(0)\left(\int_{1}^{\infty} x v(\mathrm{~d} x)+\int_{-\infty}^{-1} x v(\mathrm{~d} x)\right),
$$

and it follows that $\mathbb{E} I(t)=t \mathbb{E} V(0) \mathbb{E} \tilde{X}(1)+t \mathbb{E} V(0)\left(\int_{1}^{\infty} x v(\mathrm{~d} x)+\int_{-\infty}^{-1} x v(\mathrm{~d} x)\right)=$ $t \mathbb{E} V(0) \mathbb{E} X(1)$. Part (ii) follows from Proposition 6 (where the second term on the righthand side of (16) vanishes) and standard facts about the jump part of a Lévy process if we, for each $s \in \xi_{X}(t)$, condition on $(T(s-), V(s-))$.

The next theorem is just a combination of Theorem 1 and Proposition 8 . Note that if we take $T(t) \equiv b$, this coincides with the main result in [6].

Theorem 2. Suppose that the conditions in Proposition 8 are fulfilled. Then

$$
\begin{aligned}
t^{-1} \mathbb{E} \int_{0+}^{t} T(s) \mathrm{d} U(s)= & t^{-1} \mathbb{E}\left\{T U(t)-\int_{0+}^{t} U(s-) \mathrm{d} T(s)\right\} \\
= & \mathbb{E} V(0) \mathbb{E} X(1)+\frac{\sigma^{2}}{2} \\
& +\frac{1}{2} \int_{0}^{b} \int_{0}^{b} \pi(\mathrm{d} x, \mathrm{~d} y) \int_{-\infty}^{\infty} \varphi(x, y, z) v(\mathrm{~d} z) .
\end{aligned}
$$


Proof. The proof follows immediately from (11) and Proposition 8 if we note that, for a Lévy process, $[X, X]^{\mathrm{c}}(t)=\sigma^{2} t$.

Remark 3. At this point it may be tempting to conclude (by, e.g. mimicking the proof of part (i) of Proposition 8) that the leftmost part of (19) equals $\mathbb{E} T(0) \ell$. This is, however, not obvious. Under stationary conditions, $U$ has stationary increments (in particular, $\mathbb{E} U(t)=t \ell$ ), but it is not a Lévy process (a subordinator), so the process whose value at $t$ is $U(t)-t \ell$ need not be a martingale!

Example 3. Let $T=b$ and $X$ be a Lévy process with characteristic triplet $(\mu, \sigma, v)$ and $\mathbb{E}|X(1)|<\infty$ (this is the setup in [6]). Let $\pi$ be the stationary measure of $V$. Then in stationarity we obtain

$$
\ell=\frac{1}{b}\left(\mathbb{E} V(0) \mathbb{E} X(1)+\frac{\sigma^{2}}{2}+\frac{1}{2} \int_{0}^{b} \pi(\mathrm{d} y) \int_{-\infty}^{\infty} \varphi(b, y, z) v(\mathrm{~d} z)\right) .
$$

For examples with explicit formulae for $\pi$ and $\ell$, we refer the reader to [6].

\section{Martingales associated with the reflection}

In this section we show that the right-hand side of the general structural relationship presented in (11) is significantly simplified if we, e.g. let $X$ and $T$ be martingales which satisfy some technical conditions. More precisely, the nuisance terms $\int_{0+}^{t} V(s-) \mathrm{d} X(s)$ and $\int_{0+}^{t} U(s-) \mathrm{d} T(s)$ may in a sense be eliminated.

Lemma 1. Let $X$ be a martingale with $\mathbb{E}[X, X](t)^{2}<\infty$, and let $T$ be a downwards skip-free martingale independent of $X$ with $\mathbb{E} T(t)^{4}<\infty$ for all $t$. Then $\mathbb{E} U(t)^{2}<\infty$ and $\mathbb{E} L(t)^{2}<\infty$ for all $t \geq 0$.

Proof. By (10) and (11), it follows that

$$
2 \int_{0+}^{t} T(s) \mathrm{d} U(s)=V(0)^{2}-V(t)^{2}+2 \int_{0+}^{t} V(s-) \mathrm{d} X(s)+[X, X]^{\mathrm{c}}(t)+J_{R}(t) .
$$

By Proposition 5 we have

$$
J_{R}(t)=\sum_{s \in \xi_{X}(t)} \varphi(T(s), V(s-), \Delta X(s)) \leq \sum_{s \in \xi_{X}(t)}(\Delta X(s))^{2},
$$

so $[X, X]^{\mathrm{c}}(t)+J_{R}(t) \leq[X, X](t)$. By Theorem 29 of [19, p. 128] we know that

$$
\int_{0+}^{t} V(s-) \mathrm{d} X(s)
$$

is a local martingale. Let $T^{*}(t)=\sup _{0 \leq s \leq t} T(s)$. Plainly,

$$
\int_{0+}^{t} V(s-)^{2} \mathrm{~d}[X, X](s) \leq T^{*}(t)^{2}[X, X](t),
$$

so that

$$
\begin{aligned}
\mathbb{E} \int_{0+}^{t} V(s-)^{2} \mathrm{~d}[X, X](s) & \leq \mathbb{E} T^{*}(t)^{2}[X, X](t) \\
& =\mathbb{E} T^{*}(t)^{2} \mathbb{E}[X, X](t) \\
& \leq 4 \mathbb{E} T(t)^{2} \sqrt{\mathbb{E}[X, X](t)^{2}} \\
& <\infty .
\end{aligned}
$$


In the last step we used Doob's (maximal quadratic) inequality and the Cauchy-Schwarz inequality. In view of Corollary 3 on page 73 and Theorem 29 on page 75 of [19], (20) proves that $\int_{0+}^{t} V(s-) \mathrm{d} X(s)$ is a martingale with finite second moment for all $t$. Furthermore, by definition, $0 \leq V(0) \leq T(0)$ and $0 \leq V(t) \leq T(t)$. The above shows that

$$
\mathbb{E}\left(\int_{0+}^{t} T(s) \mathrm{d} U(s)\right)^{2}<\infty .
$$

On the other hand, we have $\int_{0+}^{t} T(s) \mathrm{d} U(s) \geq \varepsilon U(t)>0$, which shows that $\mathbb{E} U(t)^{2}<\infty$. We refrain from giving the details for $L$.

Proposition 9. Suppose that $X$ and $T$ are independent càdlàg martingales where $T$ is downwards skip-free, with $\mathbb{E}[X, X](t)^{2}<\infty$ and $\mathbb{E} T(t)^{4}<\infty$ for all $t$. Then the process $M$ defined by

$$
M(t)=\int_{0+}^{t} V(s-) \mathrm{d} X(s)+\int_{0+}^{t} U(s-) \mathrm{d} T(s)
$$

is a zero-mean martingale.

Proof. As in the proof of Lemma 1, Theorem 29 of [19, p. 128] tells us that

$$
\int_{0+}^{t} U(s-) \mathrm{d} T(s)
$$

is a local martingale and we already know that $\int_{0+}^{t} V(s-) \mathrm{d} X(s)$ is a martingale with finite second moment for all $t$. By partial integration,

$$
\int_{0+}^{t} U(s-) \mathrm{d} T(s)=T U(t)-\int_{0+}^{t} T(s-) \mathrm{d} U(s)-[U, T](t),
$$

and, thus, for each $s \leq t$,

$$
\begin{aligned}
\left|\int_{0+}^{s} U(r-) \mathrm{d} T(r)\right| & \leq|T U(s)|+\left|\int_{0+}^{s} T(r-) \mathrm{d} U(r)\right|+|[U, T](s)| \\
& \leq T^{*}(t) U(t)+T^{*}(t) U(t)+\sqrt{[U, U](t)} \sqrt{[T, T](t)} \\
& =2 T^{*}(t) U(t)+\sqrt{[U, U](t)} \sqrt{[T, T](t)},
\end{aligned}
$$

and it follows that

$$
\sup _{0 \leq s \leq t}\left|\int_{0+}^{s} U(r-) \mathrm{d} T(r)\right| \leq 2 T^{*}(t) U(t)+\sqrt{[U, U](t)} \sqrt{[T, T](t)} .
$$

By the Cauchy-Schwarz inequality, Doob's inequality, and Lemma 1, we have

$$
\mathbb{E} T^{*}(t) U(t) \leq \sqrt{\mathbb{E} T^{*}(t)^{2} \mathbb{E} U(t)^{2}} \leq 2 \sqrt{\mathbb{E} T(t)^{2} \mathbb{E} U(t)^{2}}<\infty .
$$

Since $U$ is nondecreasing, we have

$$
[U, U](t)=\sum_{0<s \leq t}(\Delta U(s))^{2} \leq \sum_{0<s \leq t} \Delta U(s)^{2} \leq U(t)^{2}
$$


and, again by the Cauchy-Schwarz inequality and Corollary 3 of $[19$, p. 73],

$$
\mathbb{E} \sqrt{[U, U](t)} \sqrt{[T, T](t)} \leq \sqrt{\mathbb{E}[U, U](t) \mathbb{E}[T, T](t)} \leq \sqrt{\mathbb{E} U(t)^{2} \mathbb{E} T(t)^{2}}<\infty
$$

If we combine (21), (22), and (23), we obtain

$$
\mathbb{E} \sup _{0 \leq s \leq t}\left|\int_{0+}^{s} U(r-) \mathrm{d} T(r)\right|<\infty
$$

which is well known to imply that the local martingale $\int_{0+}^{t} U(s-) \mathrm{d} T(s)$ is a martingale. The claim now follows by the fact that the sum of two martingales is again a martingale.

Remark 4. If $T$ is constant, it follows, by the fact that $\int_{0+}^{t} U(s-) \mathrm{d} T(s)=0$ and an inspection of the proof of Lemma 1 , that $\mathbb{E}[X, X](t)<\infty$ implies that $M$ as defined in Proposition 9 is a zero-mean martingale. It should be clear how to formulate sharper versions of Theorem 3 and Corollary 2 below corresponding to the case where $T$ is constant.

Theorem 3. Let $X$ and $T$ satisfy the conditions in Proposition 9. Then

$$
2 T U(t)=V(0)^{2}-V(t)^{2}+[X, X]^{\mathrm{c}}(t)+M(t)+J_{R}(t),
$$

where $M$ is a zero-mean martingale.

Proof. The assertion follows directly from Theorem 1 and Proposition 9.

Corollary 2. Let $X$ be a continuous martingale, and let $T$ be a downwards skip-free càdlàg martingale. Suppose that $X$ and $T$ are independent and satisfy $\mathbb{E}[X, X](t)^{2}=$ $\mathbb{E}[X, X]^{\mathrm{c}}(t)^{2}<\infty$ and $\mathbb{E} T(t)^{4}<\infty$ for all $t$. Then

$$
2 T U(t)=V(0)^{2}-V(t)^{2}+[X, X](t)+M(t),
$$

where $M$ is a zero-mean martingale.

Proof. In view of Proposition 5, $J_{R}(t)=0$. The claim now follows by Theorem 3 .

The conditions in Proposition 9 are rather strict. However, if we assume that $X$ is a Lévy process, we may prove a much sharper result. We start with a lemma.

Lemma 2. Suppose that $X$ is a Lévy process with $\mathbb{E} X(1)^{2}<\infty$ and that $\mathbb{E} V(0)^{2}<\infty$. Then $\mathbb{E} U(t)^{2}<\infty$ and $\mathbb{E} L(t)^{2}<\infty$ for all $t$.

Proof. Assume without loss of generality that $V(0)=0$. Let $V_{\varepsilon}$ and $L_{\varepsilon}$ denote the reflected process and the lower barrier local-time process corresponding to reflection at 0 and $\varepsilon>0$. In view of the assumption that $T(t)>\varepsilon$, it follows directly that $L(t) \leq L_{\varepsilon}(t)$ for all $t>0$. For $V_{\varepsilon}$, we define $\tau(1), \tau(2), \ldots$ as in the proof of Proposition 7 (with $b$ replaced by $\varepsilon$ ). We now proceed precisely as in the proof of Lemma 3.1 of [6]. By the strong Markov property $\left(V_{\varepsilon}\right.$ is strong Markov; see [6]), it follows that we may view $V_{\varepsilon}$ as regenerative with $\sigma(k), k \geq 2$, as regeneration points. Let $\sigma=\sigma(2) \wedge t$, let $N(t)$ be the number of completed cycles in $[0, t]$, and let $\Delta(t)=\left(L_{\varepsilon}(\sigma(2))-L_{\varepsilon}(\sigma(2)-)\right) \mathbf{1}_{\{\sigma(2) \leq t\}}$. Clearly, $0<\mathbb{E} \sigma^{2}<\infty$ and it follows by Proposition 6.3 of $\left[5\right.$, p. 161] that $\mathbb{E} N(t)^{2}<\infty$ for each $t$. Furthermore,

$$
\Delta(t) \leq 1 \vee \max _{0 \leq s \leq t}|\Delta X(s)| \mathbf{1}_{\{|\Delta X(s)| \geq 1\}} \leq 1+\sum_{0 \leq s \leq t}|\Delta X(s)| \mathbf{1}_{\{|\Delta X(s)| \geq 1\}},
$$


and $\sum_{0 \leq s \leq t}|\Delta X(s)| \mathbf{1}_{\{|\Delta X(s)| \geq 1\}}$ is a subordinator with finite second moment (because $\left.\mathbb{E} X(1)^{2}<\infty\right)$, which implies that $\mathbb{E} \Delta(t)^{2}<\infty$. Let $m_{\varepsilon}(t)=\sup _{0 \leq s \leq t}(-X(s))$ denote the local time of the process, which is one-sided reflected at 0 . If we write $-X(t)=$ $-X(t)+t \mathbb{E} X(1)-t \mathbb{E} X(1)$, we see that $-X(t)$ is the sum of a locally square-integrable martingale and a deterministic function, and it follows by Doob's inequality that $\mathbb{E} m_{\varepsilon}(t)^{2}<\infty$. It follows by Wald's second moment identity, see [5, p. 415], that $\mathbb{E}\left(L_{\varepsilon}(t)-\mu N(t)\right)^{2}<\infty$, where $\mu=\mathbb{E}\left(m_{\varepsilon}(t)+\Delta(t)\right)$. Thus, $L_{\varepsilon}(t)=\left(L_{\varepsilon}(t)-\mu N(t)\right)+\mu N(t)$ has finite second moment and so has $L(t)$. Finally, it follows by (3) that

$$
U(t) \leq U(t)+V(t)=V(0)+X(t)+L(t)
$$

which shows that $\mathbb{E} U(t)^{2}<\infty$.

Theorem 4. Let $X$ be a Lévy process with $\mathbb{E} X(1)^{2}<\infty$, and let $T$ be an independent càdlàg martingale with $\mathbb{E} T(t)^{2}<\infty$ for all $t$. Then

$$
2 T U(t)=V(0)^{2}-V(t)^{2}+2 \mathbb{E} X(1) \int_{0}^{t} V(s-) \mathrm{d} s+\sigma^{2} t+M(t)+J_{R}(t),
$$

where $M$ is a zero-mean martingale.

Proof. We write $X(t)=X(t)-t \mathbb{E} X(1)+t \mathbb{E} X(1)$ and note that $M_{X}(t)=X(t)-t \mathbb{E} X(1)$ defines a martingale. Then

$$
\int_{0+}^{t} V(s-) \mathrm{d} X(s)=\int_{0+}^{t} V(s-) \mathrm{d} M_{X}(s)+\mathbb{E} X(1) \int_{0+}^{t} V(s-) \mathrm{d} s .
$$

By precisely the same arguments as in the proof of Lemma 1, we show that $\int_{0+}^{t} V(s-) \mathrm{d} M_{X}(s)$ is a martingale (note that $\mathbb{E} M_{X}(t)^{2}=\mathbb{E}\left[M_{X}, M_{X}\right](t)$; see Corollary 3 of $[19$, p. 73]). That $\int_{0+}^{t} U(s-) \mathrm{d} T(s)$ is a martingale follows by the proof of Proposition 9 where $\mathbb{E} U(t)^{2}<\infty$ follows by Lemma 2 . We complete the proof by applying Theorem 1.

Example 4. Let $X$ be Brownian motion with variance $\sigma^{2}$ and drift $\mu$, and let $Z$ be a downwards skip-free Lévy process which is independent of $X$. For simplicity, we assume that $\Delta Z(t) \leq c$ for some constant $c$, so that all exponential moments of $Z$ exist. Take $W$ to be the Wald martingale corresponding to $Z$, i.e.

$$
W(t)=\mathrm{e}^{\alpha Z(t)-t \kappa(\alpha)}, \quad \alpha>0,
$$

where $\kappa(\cdot)$ denotes the Lévy exponent of $Z, 0<\varepsilon<1$, and

$$
\tau=\inf \{t>0: \alpha Z(t)-t \kappa(\alpha)=\log \varepsilon\}
$$

and let $T(t)=W(t \wedge \tau)$. Then, clearly, the conditions of Theorem 4 are fulfilled, and, thus,

$$
U(t)=\frac{\mathrm{e}^{-\alpha Z(t \wedge \tau)+(t \wedge \tau) \kappa(\alpha)}}{2}\left\{V(0)^{2}-V(t)^{2}+2 \mu \int_{0}^{t} V(s) \mathrm{d} s+\sigma^{2} t+M(t)\right\},
$$

where $M$ is a zero-mean martingale. Note that in (25), $U$ and $V$ are continuous. 
Example 5. Take $X$ to be a continuous martingale such that $\mathbb{E}[X, X](t)<\infty$ and $[X, X](t) \stackrel{\text { a.s. }}{\rightarrow} \infty$ as $t \rightarrow \infty$, let $T=b>0$, and let $\tau_{a}=\inf \{t>0:[X, X](t)>a\}, a>0$. If we apply the version of Corollary 2 corresponding to constant $T$ and take expectations at $t=\tau_{a}$, we obtain

$$
2 b \mathbb{E} U\left(\tau_{a}\right)=\mathbb{E} V(0)^{2}-\mathbb{E} V\left(\tau_{a}\right)^{2}+a+\mathbb{E} M\left(\tau_{a}\right) .
$$

By optional stopping, $M\left(t \wedge \tau_{a}\right)=\int_{0}^{t} V(s) \mathbf{1}_{\left\{\tau_{a} \geq s\right\}} \mathrm{d} X(s)$ is a zero-mean martingale and it holds that $\mathbb{E} M\left(t \wedge \tau_{a}\right)^{2} \leq b^{2} a$. By Doob's inequality, we obtain

$$
\mathbb{E}\left(\sup _{0 \leq s \leq t}\left|M\left(s \wedge \tau_{a}\right)\right|\right)^{2} \leq 4 \mathbb{E} M\left(t \wedge \tau_{a}\right)^{2} \leq 4 b^{2} a,
$$

and, if we let $t \rightarrow \infty$ in (27), it follows by monotone convergence that

$$
\mathbb{E}\left(\sup _{s \leq \tau_{a}}|M(s)|\right)^{2}<\infty .
$$

We bound $\left|M\left(t \wedge \tau_{a}\right)\right|$ by $\sup _{s \leq \tau_{a}}|M(s)|$, which has finite expectation in view of (28). It now follows by dominated convergence that

$$
\mathbb{E} M\left(\tau_{a}\right)=\lim _{t \rightarrow \infty} \mathbb{E} M\left(t \wedge \tau_{a}\right)=0,
$$

and from (26) we obtain the formula $2 b \mathbb{E} U\left(\tau_{a}\right)=\mathbb{E} V(0)^{2}-\mathbb{E} V\left(\tau_{a}\right)^{2}+a$. Since $U\left(\tau_{a}\right) \geq 0$, we obtain the bound

$$
\mathbb{E} V\left(\tau_{a}\right)^{2} \leq a+\mathbb{E} V(0)^{2}
$$

which is nontrivial for $a<b^{2}-\mathbb{E} V(0)^{2}$. If $[X, X](t)$ is deterministic, equal to $f(t)$, say, and we take $V(0)=0$, we obtain $\mathbb{E} V(t)^{2} \leq f(t)$ for $t \leq \inf \left\{s>0: f(s)>b^{2}\right\}$.

Example 6. Let $X$ be a Lévy process with finite second moment, and let $T=b$. We assert that $U(t) / t \stackrel{\text { a.s. }}{\rightarrow} \ell$ as $t \rightarrow \infty$. To prove this we will use a well-known ad-hoc method. It holds that $[X, X](t)=\sigma^{2} t+\tilde{X}(t)$, where $\tilde{X}$ is a subordinator with finite first moment, which we denote by $\tilde{\mu}$. Thus, we may write

$$
[X, X](t)=\left(\sigma^{2}+\tilde{\mu}\right) t+\tilde{M}(t),
$$

where $\tilde{M}(t)=\tilde{X}(t)-\tilde{\mu} t$ is a zero-mean martingale. Take $M$ to be as in Theorem 4. Let $\hat{M}(t)=\int_{0}^{t}(1+s)^{-1} \mathrm{~d} M(s)$. Then $\hat{M}$ is a local martingale and further

$$
\begin{aligned}
{[\hat{M}, \hat{M}](t) } & =\int_{0}^{t}(1+s)^{-2} \mathrm{~d}[M, M](s) \\
& =\int_{0}^{t}(1+s)^{-2} V(s-)^{2} \mathrm{~d}[X, X](s) \\
& =\int_{0}^{t}(1+s)^{-2} V(s-)^{2}\left(\sigma^{2}+\tilde{\mu}\right) \mathrm{d} s+\int_{0}^{t}(1+s)^{-2} V(s-)^{2} \mathrm{~d} \tilde{M}(s) .
\end{aligned}
$$

The part $\int_{0}^{t}(1+s)^{-2} V(s-)^{2} \mathrm{~d} \tilde{M}(s)$ is a zero-mean martingale (and not just a local martingale). This follows since $\sup _{0 \leq s \leq t}\left|\int_{0}^{s}(1+u)^{-2} V(u-)^{2} \mathrm{~d} \tilde{M}(u)\right|$ has finite mean (this is straightforward to show). Furthermore,

$$
\int_{0}^{t}(1+s)^{-2} V(s-)^{2}\left(\sigma^{2}+\tilde{\mu}\right) \mathrm{d} s \leq\left(\sigma^{2}+\tilde{\mu}\right) b^{2} .
$$


Thus, $\mathbb{E}[\hat{M}, \hat{M}](t) \leq\left(\sigma^{2}+\tilde{\mu}\right) b^{2}$ and it follows that $\hat{M}$ is a martingale with $\mathbb{E} \hat{M}(t)^{2}=$ $\mathbb{E}[\hat{M}, \hat{M}](t) \leq\left(\sigma^{2}+\tilde{\mu}\right) b^{2}$. By standard results on martingale convergence, this shows that $\lim _{t \rightarrow \infty} \hat{M}(t)$ exists and is finite a.s. By partial integration in the definition of $\hat{M}(t)$, we obtain $M(t) / t \stackrel{\text { a.s. }}{\rightarrow} 0$ as $t \rightarrow \infty$. We divide both sides of (24) by $t$ and let $t \rightarrow \infty$. The assertion follows by the regenerative structure of $V$ and the stationary independent increments of $X$.

\section{Acknowledgement}

The authors wish to thank an anonymous referee for pointing out two mistakes in the submitted manuscript as well as for valuable suggestions which improved the final version of the paper.

\section{References}

[1] Andersen, L. N. (2011). Subexponential loss rate asymptotics for Lévy processes. Math. Meth. Operat. Res. 73, 91-108.

[2] Andersen, L. N. And Asmussen, S. (2011). Local time asymptotics for centered Lévy processes with two-sided reflection. Stoch. Models 27, 202-219.

[3] Andersen, L. N. And MandJes, M. (2009). Structural properties of reflected Lévy processes. Queueing Systems 63, 301-322.

[4] Asmussen, S. (1995). Stationary distributions via first passage times. In Advances in Queueing, ed. J. Dshalalow, CRC, Boca Raton, FL, pp. 79-102.

[5] Asmussen, S. (2003). Applied Probability and Queues, 2nd edn. Springer, New York.

[6] Asmussen, S. And Pihlsgård, M. (2007). Loss rates for Lévy processes with two reflecting barriers. Math. Operat. Res. 32, 308-321.

[7] BeKKer, R. AND Zwart, B. (2005). On an equivalence between loss rates and cycle maxima in queues and dams. Prob. Eng. Inf. Sci. 19, 241-255.

[8] Billingsley, P. (1999). Convergence of Probability Measures, 2nd edn. John Wiley, New York.

[9] Cooper, W. L., Schmidt, V. And Serfozo, R. F. (2001). Skorohod-Loynes characterizations of queueing, fluid, and inventory processes. Queueing Systems 37, 233-257.

[10] D'Auria, B. AND Kella, O. (2012). Markov modulation of a two-sided reflected Brownian motion with application to fluid queues. Stoch. Process. Appl. 122, 1566-1581.

[11] Jelenković, P. R. (1999). Subexponential loss rates in a GI/GI/1 queue with applications. Queueing Systems 33, 91-123.

[12] Kella, O. (2006). Reflecting thoughts. Statist. Prob. Lett. 76, 1808-1811.

[13] Kella, O., Boxma, O. And Mandjes, M. (2006). A Lévy process reflected at a Poisson age process. J. Appl. Prob. 43, 221-230.

[14] Kim, H. S. AND Shroff, N. B. (2001). On the asymptotic relationship between the overflow probability and the loss ratio. J. Appl. Prob. 33, 836-863.

[15] Kruk, L., Lehoczky, J., Ramanan, K. and Shreve, S. (2007). An explicit formula for the Skorokhod map on [0, a]. Ann. Prob. 35, 1740-1768.

[16] Moran, P. A. P. (1959). The Theory of Storage. Methuen, London.

[17] Palmowski, Z. And ŚwiA̧TeK, P. (2011). Loss rate for a general Lévy process with downward periodic barrier. In New Frontiers in Applied Probability (J. Appl. Prob. Spec. Vol. 48A), eds P. Glynn, T. Mikosch and T. Rolski, Applied Probability Trust, Sheffield, pp. 99-108.

[18] Pihlsgård, M. (2005). Loss rate asymptotics in a $G I / G / 1$ queue with finite buffer. Stoch. Models 21,913-931.

[19] Protter, P. E. (2004). Stochastic Integration and Differential Equations, 2nd edn. Springer, Berlin.

[20] Sato, K.-I. (1999). Lévy Processes and Infinitely Divisible Distributions. Cambridge University Press.

[21] Stadje, W. (1993). A new look at the Moran dam. J. Appl. Prob. 30, 489-495.

[22] Zwart, A. P. (2000). A fluid queue with a finite buffer and subexponential input. Ann. Prob. 32, 221-243. 